\title{
Digital Eyewear in Cardiology-Looking Forward
}

\section{Stephen J Leslie ${ }^{1,2 *}$ and Charles Knoery ${ }^{1}$}

\section{${ }^{1}$ Cardiac Unit, Raigmore Hospital, Inverness, United Kingdom \\ ${ }^{2}$ Department of Diabetes \& Cardiovascular Science, Centre for Health Science, University of the Highlands and Islands, Inverness, United Kingdom}

\author{
Keywords \\ Wearable technology, Cardiology, Telehealth, Augmented reality
}

Abbreviations

AR: Augmented reality; ECG: Electrocardiogram

\section{Text}

Cardiovascular disease (CVD) remains a major cause of morbidity and mortality worldwide [1] and consumes a large proportion of health care budgets due to acute admissions to hospital, hospital transfers, investigations, treatment and outpatient follow up. Managing CVD in remote areas may be particularly challenging (and expensive) as there are additional geographical barriers to patients gaining specialist care and advice. This has a led to a feeling that the current situation is not sustainable and that we must change or adapt the way we provide healthcare.

Over the last 30 years, the use of established technologies (telephone, mobile phone, e-mail and internet) has greatly enhanced communication not only between health care providers [2,3] but also with patients $[4,5]$, and their use is increasing. More recently, technology has been hailed as a potential solution to the health care crisis, for example, by facilitating enhanced self-management in patients with chronic conditions such as heart failure. While heavily supported by governmental policy and guidelines, technology enhanced care has so far failed to find a significant role in routine clinical practice. For example, despite a lack of consistent evidence, home based tele-monitoring for patients with chronic heart failure has featured in several UK government policy documents. There is a danger that this becomes a 'technology driven environment' rather than 'clinically driven environment' i.e. new technology is looking for a clinical problem to solve rather than a clinical problem looking for the best solution (which may include technology). This situation risks 'technology' losing credibility with clinical staff.

The concept of wearable computing has been around for some time but it is the recent confluence of cloud based data storage and analysis, high speed wireless connectivity and smartphone technology that has led to an expansion of wearable technology (e.g. fitness bands and digital eyewear). Fitness bands (fit bits) have been successful in the commercial markets but slower to be embraced in healthcare. There are several potential reasons for this. Generally healthcare providers are fiscally constrained and new interventions will compete with established treatments for resource. In addition, clinical staff tends to be conservative and demand a high level of evidence before using new therapies and interventions. Therefore, speculative new technology, no matter how cheap or impressive, may be viewed as 'high risk'. Digital eyewear is a more recent development. In 2014 google glass was launched to the public, but quickly withdrawn from a market that appeared not ready for this development mainly due to public concerns about privacy and safety. Despite this, development of wearable technology continues and a range of digital eyewear devices are now available. In clinical medicine, and cardiology in particular, there is a need for clinical assessment and expert knowledge combined with visualisation and interpretation of test results (angiograms, ECGs, echocardiography). These clinical situations make digital eyewear a potentially useful professional tool to allow data to be brought closer to the patient-cardiologist interface or integrated into existing clinical procedures.

The future clinical uses of digital eyewear can be broadly divided into two areas; 'receiving data' and 'creating data'. Within 'receiving data', augmented reality (AR, superimposing an image on the view of the real world providing a composite view) is potentially the most exciting application of digital eye wear. AR has a wide range of potential uses including enhancing medical procedures to improving training [6] and even ongoing appraisal of clinician skills (e.g. cath lab skills, cardiac arrest scenarios etc). Medical training has always had a

*Corresponding author: Stephen J Leslie, Professor,
Consultant Cardiologist, Highland Heartbeat Centre,
Cardiac Unit, Raigmore Hospital Inverness, IV2 3UJ, United
Kingdom, Tel: +44 1463 705459/62/+44 1463705460 ,
E-mail: stephen.leslie@nhs.net
Received: September 16, 2016: Accepted: October 08,
2016: Published online: October 10, 2016
Citation: Leslie SJ, Knoery C (2016) Digital Eyewear in
Cardiology-Looking Forward. Scientific Pages Heart 1:003e.

Copyright: () 2016 Leslie SJ, et al. This is an open-access article distributed under the terms of the Creative Commons Attribution License, which permits unrestricted use, distribution, and reproduction in any medium, provided the original author and source are credited. 
large degree of apprenticeship. Digital eyewear has the potential to enable 'over the shoulder' support without the need for co-location, which is a huge benefit in remote and rural settings or for low frequency complex procedure. The 'sexier' aspects of digital eyewear such as overlaying 3D imaging onto a patient using $\mathrm{AR}$ are getting much of the attention (and funding), but the more simple use cases in terms of receiving and generating very basic data can be applied to solve everyday problems using existing technology. Simply 'viewing data' via digital eyewear could bring data closer to the patientcardiologist interface. Early research into using digital eye wear to view chest X-rays and ECG have shown that this is possible but that visualisation has less fidelity compared to computer screens or mobile phones $[7,8]$. An upgrade with hand free zoom and pan technology did seem to improve this functionality [9] but more development is required.

'Creating data' at the point of care also has several potential applications. For instance, in remote area, patients could be included in multidisciplinary team meetings or virtual clinics before potentially long or dangerous inter-hospital transfers. Creating visual records of a patient's functional abilities may allow clinicians to better assess referrals, particularly in elderly patients and better decide on the appropriate treatment. Such technology might also be used to support clinical staff (e.g. community based heart failure nurses whether admission to hospital is required for patients at home). One study used google glass to record coronary angiograms for remote display on an ipad and desk top [10]. Whether digital eye wear used for the above purpose will result in better care or more appropriate decision making is currently unknown. The largest potential barrier to widespread adoption is the huge concern around privacy and the handling of patient confidential data. Solutions to this need to be developed alongside establishing the clinical benefits.

What is certain is that there are an increasing number of digital 'solutions'. However, to determine if these will improve outcomes for patients will require high quality clinical trials. Industry, academia, clinicians and patient must work together for this to happen.
In summary, the array of new technology is sleek, tactile and the functionality beguiling.... cardiologists may want it ..... but do our patients need it?

\section{Acknowledgments}

The authors would like to thank Chris Bryson (summit wearables) and Alan Whiteside (NHS Highland) for their guidance in preparing this editorial.

\section{References}

1. WHO global status report on non-communicable disease as a potential reference?.

2. Barclay JL, MacFarlane P, Potts S, et al. (2008) Evaluation of a new device for the transmission of electrocardiograms by e-mail. J Telehealth Telecare 14: $219-220$.

3. Rushworth GF, Bloe C, Diack HL, et al. (2014) Pre-hospital ECG E-transmission for patients with suspected myocardial infarction in the highlands of Scotland. Int J Environ Res Public Health 11: 2346-2360.

4. Gandiya T, Dua A, King G, et al. (2012) Self-reported "communication technology" usage in patients attending a cardiology outpatient clinic in a remote regional hospital. Telemed J E Health 18: 219-224.

5. Jones J, Cassie S, Thompson M, et al. (2014) Delivering healthcare information via the internet: cardiac patients' access, usage, perceptions of usefulness, and web site content preferences. Telemed J E Health 20: 223-227.

6. Knight HM, Gajendragadkar PR, Bokhari A (2015) Wearable technology: Using Google Glass as a teaching tool. BMJ Case Rep 2015.

7. Jeroudi OM, Christakopoulos G, Christopoulos G, et al. (2015) Accuracy of remote electrocardiogram interpretation with the use of Google Glass technology. Am J Cardiol 115: 374-377.

8. Spaedy E, Christakopoulos GE, Tarar MN, et al. (2016) Accuracy of remote chest X-ray interpretation using Google Glass technology. Int J Cardiol 219: 38-40.

9. Stetler J, Resendes E, Martinez-Parachini JR, et al. (2016) Hands-free zoom and pan technology improves the accuracy of remote electrocardiogram interpretation using Google Glass. Int J Cardiol 204: 147-148.

10. Duong T, Wosik J, Christakopoulos GE, et al. (2015) Interpretation of Coronary Angiograms Recorded Using Google Glass: A Comparative Analysis. J Inv Cardiol 27: 443-446. 